\title{
Xeroradiographic techniques applied to assessment of Achilles tendon in inflammatory or metabolic diseases
}

\author{
J. C. GERSTER, H. HAUSER, AND G. H. FALLET \\ From the Department of Medicine, Division of Rheumatology and Department of Radiology, \\ University Hospital, Geneva, Switzerland
}

\begin{abstract}
Gerster, J. C., Hauser, H., and Fallet, G. H. (1975). Annals of the Rheumatic Diseases, 34, 479-488. Xeroradiographic techniques applied to assessment of Achilles tendon in inflammatory or metabolic diseases. Ten patients with inflammatory disease(rheumatoid arthritis, ankylosing spondylitis, Reiter's disease) or metabolic disease (gout, pseudogout, tendinous xanthomatosis) affecting the Achilles tendons are presented and discussed. Radiological lateral views of heel were obtained with xeroradiographic techniques, which permitted the recording on the same image of details of both bone and soft tissue and the evaluation and quantitation of the changes in the Achilles tendons. Xeroradiography seems to be a very suitable radiological technique for routine use in the evaluation and follow up of rheumatic diseases of the foot.
\end{abstract}

It is often difficult to evaluate and quantitate lesions affecting the soft tissues around bones and joints, especially if there is associated obesity. However, xeroradiography is useful in the radiological assessment of such lesions because the soft tissues as well as the bony structure can be recorded in detail on the same exposure. Xeroradiography is a technique using photoconductors and electrostatic charges as a method of recording $x$-ray images (Oliphant, 1955; Wolfe, 1969).

We present here the advantages of this method as used with ten patients suffering from various lesions of the heel and the Achilles tendon.

\section{Methods}

A Rank Xerox 125 system was used. Xeroradiographs of the foot were obtained with high $\mathrm{kV}(120 \mathrm{kV})$ and low $\mathrm{mA}(6-10 \mathrm{~mA})$. The distance between the plate and the tube was $1.0 \mathrm{~m}$. A slight underexposure was chosen in order to produce a better contrast of the images. Positive pictures were recorded. The average radiation dose received by the patient in this study was $140 \mathrm{mrad}$ or $160 \mathrm{mR}$.

Normal mean diameters of the Achilles tendon were determined in 15 adult subjects with generalized osteoarthrosis without pathological changes of the heels.

\section{Normal findings}

A normal lateral xeroradiograph of the heel is explained in detail in Fig. 1. The mean anteroposterior diameter of the Achilles tendon was measured at the level of the subAchilles bursa, on original xeroradiograph as $5 \cdot 1 \pm 1.0 \mathrm{~mm}$ (SD). It was also determined at the middle part of the tendon between the calcanean and gastrocnemial attachments and was $6 \cdot 2 \pm 1 \cdot 6 \mathrm{~mm}(\mathrm{SD})$.

\section{Clinical features}

Lesions of the heel often occur in Reiter's disease and ankylosing spondylitis. The inflammatory process begins in the soft tissues (Achilles peritendinitis, plantar fasciitis) and later changes of the calcanean bone can develop (spurs, erosions, periosteal new bone formation). This is illustrated in the following 2 cases.

Case 1, male, aged 42, had Reiter's disease for 7 years. He occasionally felt pain in the left heel, which, during the past 3 years, had localized at the insertion of the right Achilles tendon; it was swollen and tender to pressure. Xeroradiography (Fig. 2) showed both calcanei to be normal; the Achilles tendons were diffusely thickened, especially the left one.

Case 2, male, aged 39, with HL-A 27 and ankylosing spondylitis for 3 years, had pain on both the plantar and 

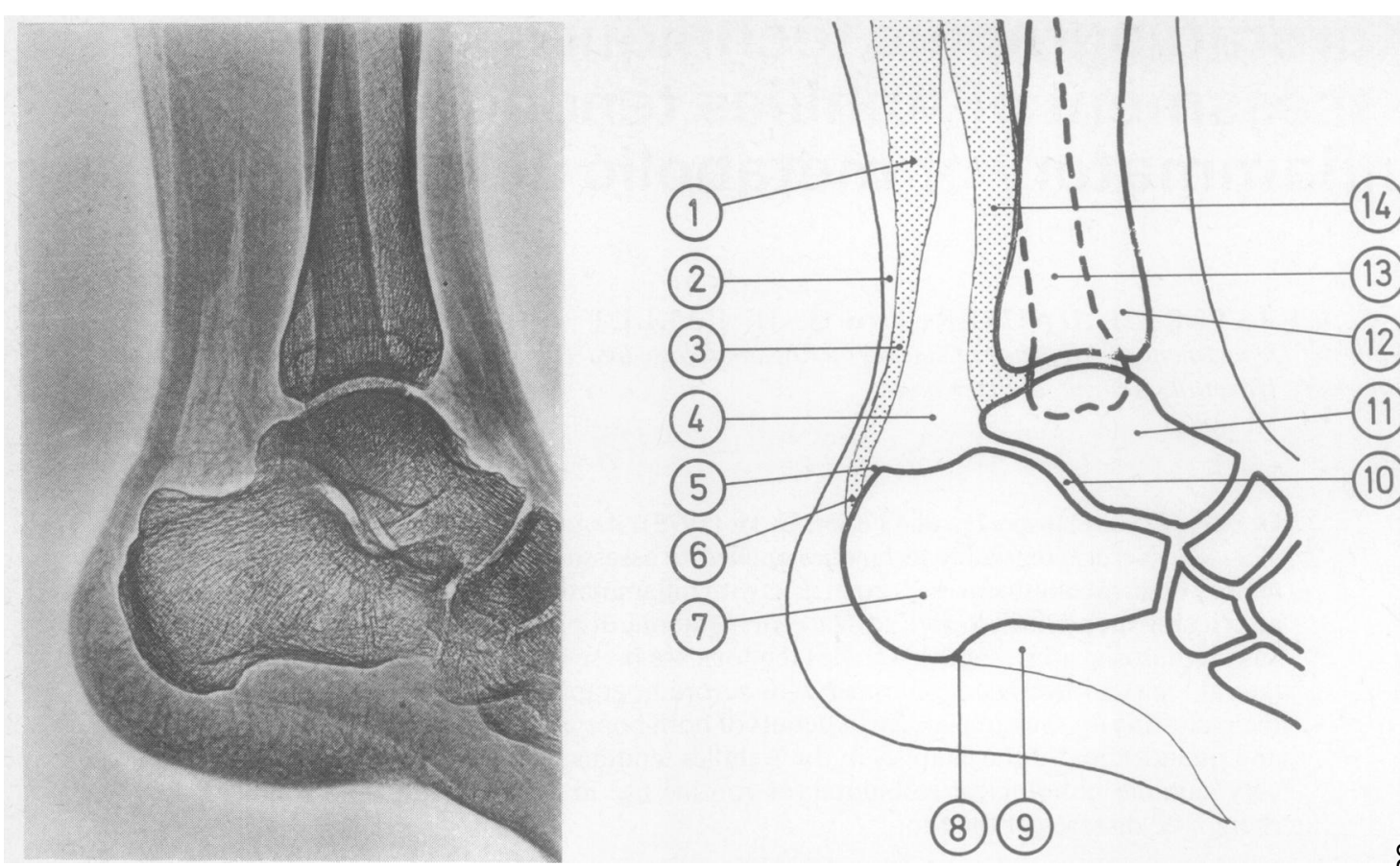

ᄋ $\overrightarrow{0}$

FIG. 1 Lateral view of the right heel. Normal xeroradiographic image. 1. Gastrocnemius muscle; 2. skin; 3. Achilles or tendon; 4. Kagers's triangle (triangular space filled with fatty tissue); 5. sub-Achilles bursa; 6. insertion of Achilles tendōi on calcaneum; 7. calcaneum; 8. insertion of plantar aponeurosis on calcaneum; 9. flexor digitorum brevis muscle; 10. sub talar joint; 11. talus; 12. tibia; 13. fibula; 14. deep flexors of the calf

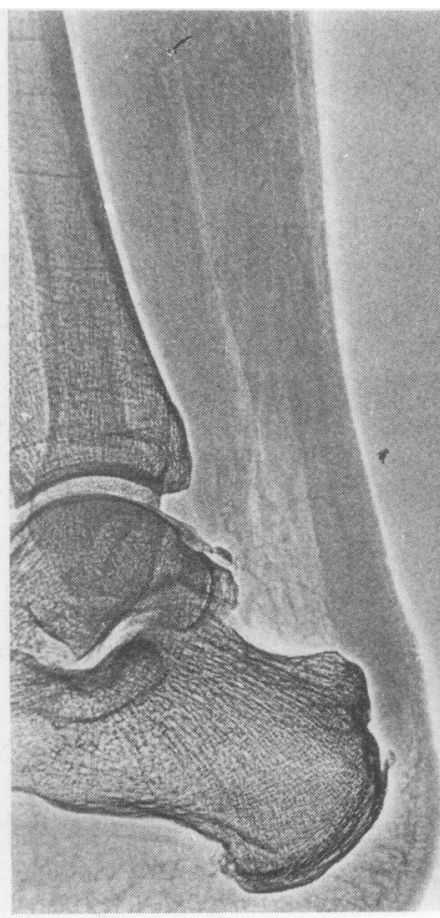

(L)

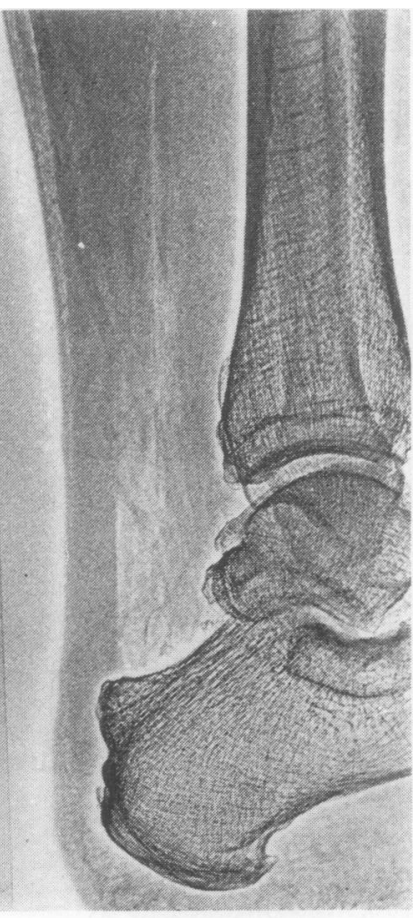

(R)
FIG. 2 Reiter's disease (Case 1). Bilateral diffuse thickening of Achilles tendons; 으 calcifications at the insertion of the tendons. Inferior diameter of right $8.0 \mathrm{~mm}$, left 13.0 . $\mathrm{mm}$; diameter at middle part of right $8.0 \mathrm{~mm}$, ठิ left $10.0 \mathrm{~mm}$ 


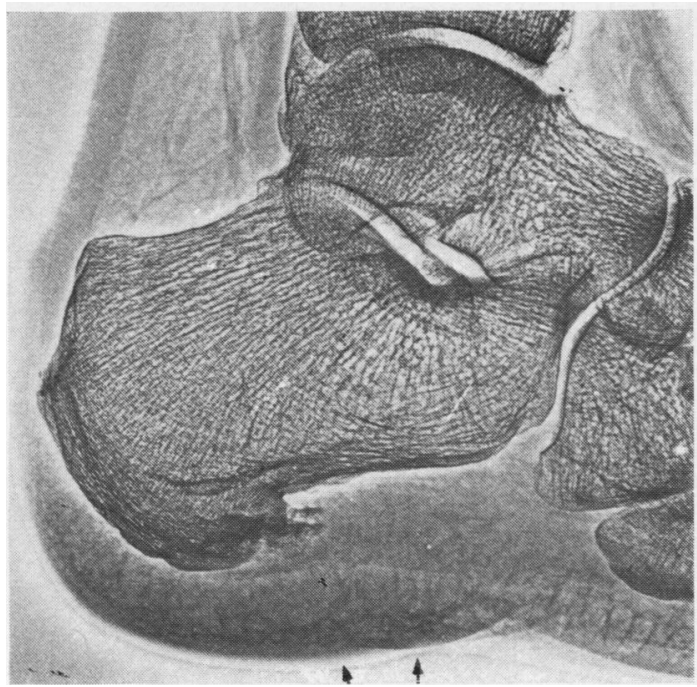

FIG. 3 Ankylosing spondylitis (Case 2). Right heel. Achilles tendon is normal; thickened heel pad (arrows); the overgrowth measures $25 \mathrm{~mm}$ on the original xeroradiograph (22 $\mathrm{mm}$ on the left, normal heel). Note the irregular plantar spur projecting into the plantar aponeurosis and into the insertion of the flexor digitorum brevis muscle
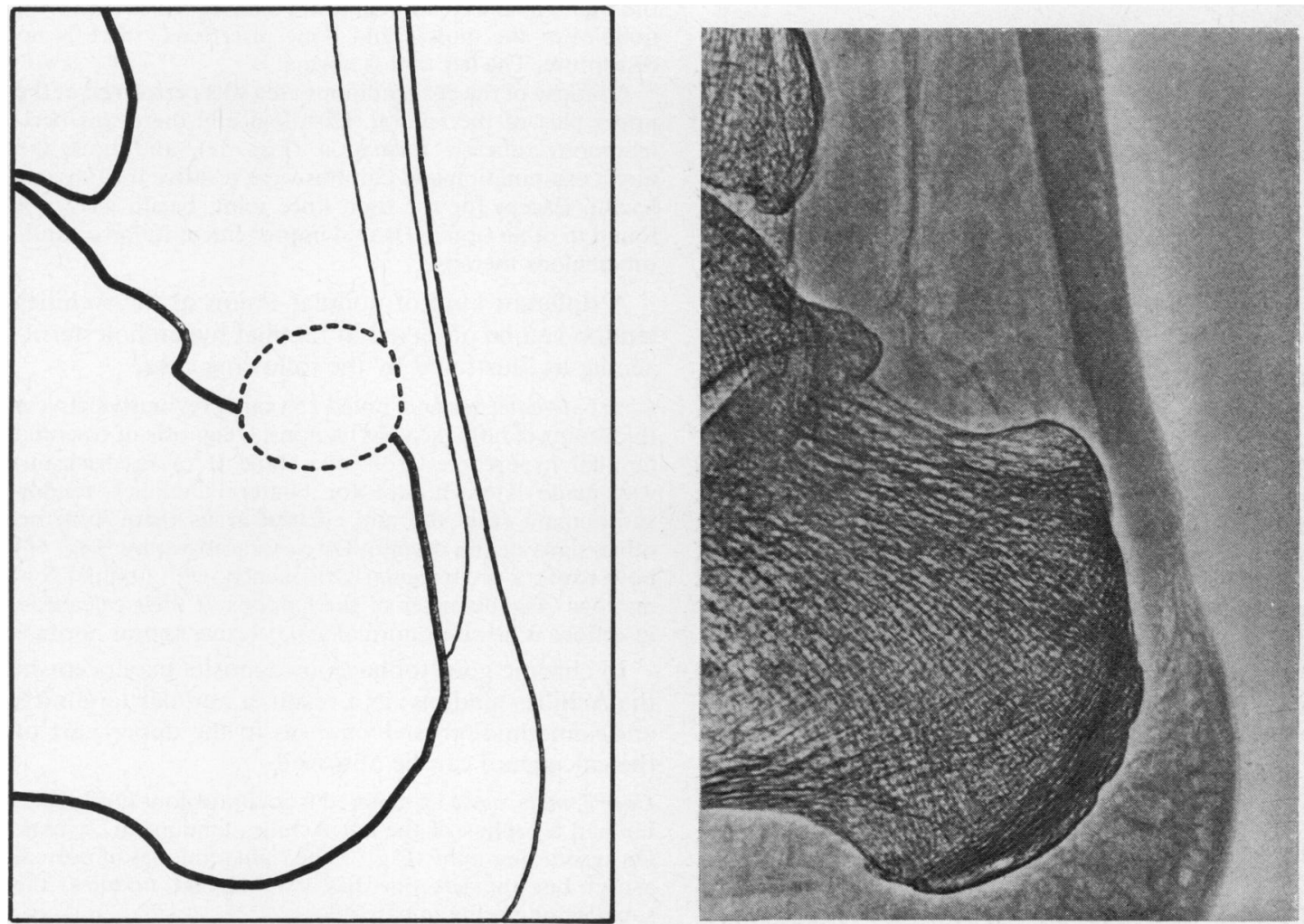

(a)

FIG. 4(a) Rheumatoid arthritis (Case 3). Rounded clarity in area of the pre-Achilles bursa with a discrete impression on the anterior part of the Achilles tendon. This image is considered to be that of an active pre-Achilles bursitis. Diagram: thick line = calcanean surface; thin line = limits of Achilles tendon; broken line = enlarged pre-Achilles bursa 


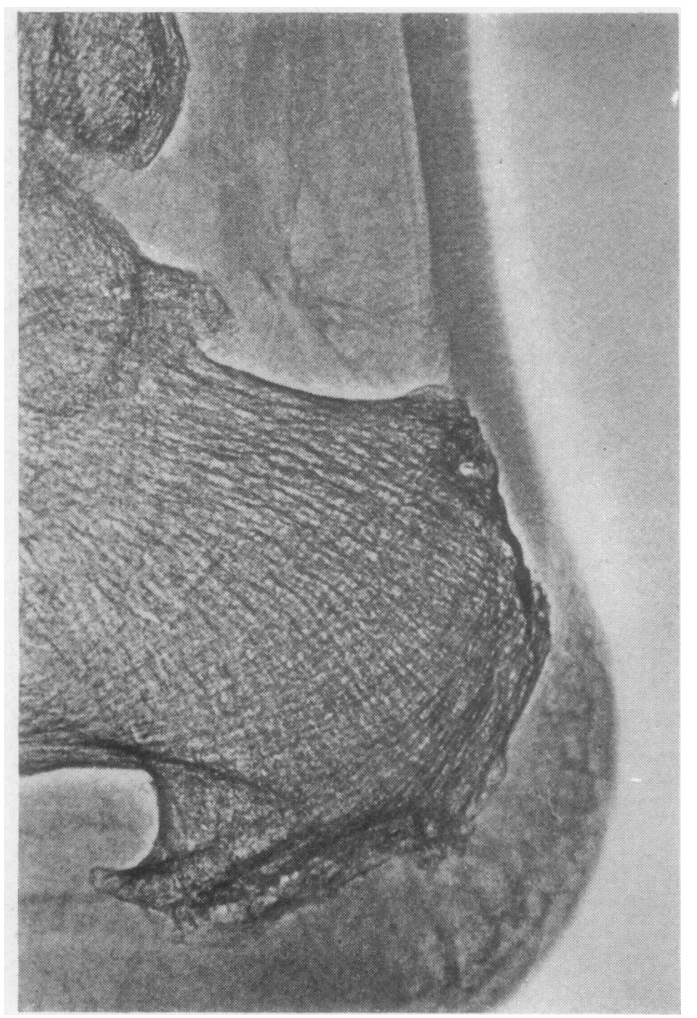

FIG. 4(b) Rheumatoid arthritis (Case 4). Sub-Achilles healed calcaneal erosions with remineralization. Irregular scars on the anterior aspect of the tendon adjacent to the pre-Achilles bursa. Plantar spur; periosteal new bone formation at the posterior aspect of the calcaneus

posterior aspects of the right heel. There was acute tenderness on pressure over the os calcis, at the insertion of the Achilles tendon and of the plantar aponeurosis. Xeroradiography (Fig. 3) failed to show any tendinous thickening or irregularity; however, the heel pad was clearly thickened and a large fluffy subplantar spur was present. Xeroradiography permitted the elimination of a diagnosis of achillotendinitis or achillobursitis, but showed inflammation of the plantar pad.

In rheumatoid arthritis (RA) the sub-Achilles bursa is sometimes inflamed, which occasionally leads to erosions of the upper calcaneus.

Case 3, female, aged 62, had RA in the hands, knees, and ankles for 5 years. Recent examination showed tenderness over both Achilles tendons. The left one appeared swollen near its insertion and a fluctuant mass was felt protruding on both sides of the tendon. An attempt to withdraw fluid by needle aspiration was unsuccessful. Conventional $x$-rays of the heels were normal, whereas on the xeroradiograph there was definite clarity in the area of the left preAchilles bursa and an impression on the anterior part of the Achilles tendon (Fig. 4a). This image was interpreted as that of an active achillobursitis.
Case 4, female, aged 61, with a history of severe, nodular seropositive RA of 20 years' duration, had tenderness on pressure of the posterior aspect of the right calcaneus at the attachment of the Achilles tendon. On xeroradiography (Fig. $4 b$ ) the changes in the calcanean bone are seen; healed erosions adjacent to the Achilles bursa are seen, as well as plantar spurs and periosteal new bone formation at the posterior part of the calcaneus. There is no thickening of the Achilles tendon but irregular anterior scars are found at the level of the pre-Achilles bursa, probably representing the residual signs of a healed achillobursitis.

Tuberculous tenosynovitis is uncommon at the present time, but was sometimes diagnosed before antituberculous therapy became common. The diagnosis should still be considered in the presence of a nodular tendinitis.

Case 5, male, aged 63, had received prednisone and azathioprine for 14 months because of a subacute polymyositis. He suddenly developed severe pain in the right knee and the right calf, accompanied by subfebrile temperature and loss of appetite. The right knee and Achilles tendon were warm and swollen (Fig. 5a). The tendon was tender to pressure and the dorsiflexion of the ankle was limited by pain. On xeroradiograph (Fig. $5 b$ ) the right Achilles tendon appears diffusely thickened with nodules at the muscle and bone insertions; there is no disrupture. The left foot is normal.

A biopsy of the peritendinous area was performed at the upper part of the tendon. Histologically there was perio tendinous tubercle formation (Fig. 5c), and both the direct examination and cultures were positive for tubercle bacilli. Except for the right knee joint, bacilli were not found in other organs. Rapid improvement followed antituberculous therapy.

A different kind of nodular lesion of the Achilles tendon can be observed in familial hypercholesterolaemia, as illustrated by the following case.

Case 6, female, aged 68, noted 15 years previously painless thickening of both Achilles tendons. Diagnosis of essential familial hypercholesterolaemia (type II of Fredrickson) was made later. Except for bilateral Achilles tendon xanthomata (Fig. 6a) and corneal arcus there were no other signs of the disease. On xeroradiography (Fig. $6 b$ ) both tendons are irregularly thickened, with nodular formations. The diameter of the tendons at their calcanean insertions is however normal and calcanei appear normal.

In chronic gout tophaceous deposits may occur in the Achilles tendons; as a result, a nodular tendinitis and sometimes erosive changes in the upper part of the calcaneum can be observed.

Case 7, male, aged 53, suffered from urate gout for 8 years. $\mathrm{He}$ had a tophus of the left Achilles tendon for 2 years. On xeroradiography (Fig. 7), the right tendon is of normal aspect but the left one has two distinct nodules, the superior one being impalpable.

Case 8 , male, aged 52, had a history of gouty arthritis of both feet for 7 years, a tophus appears in the right Achilles tendon 2 years ago (Fig. 8a); it was tender to pressure. Xeroradiography (Fig. $8 b$ ) gives an excellent image of the tophus. 


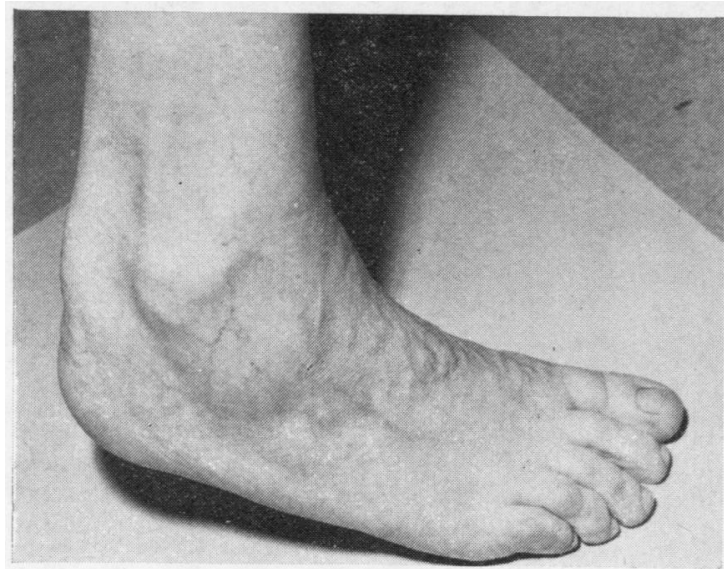

(a)

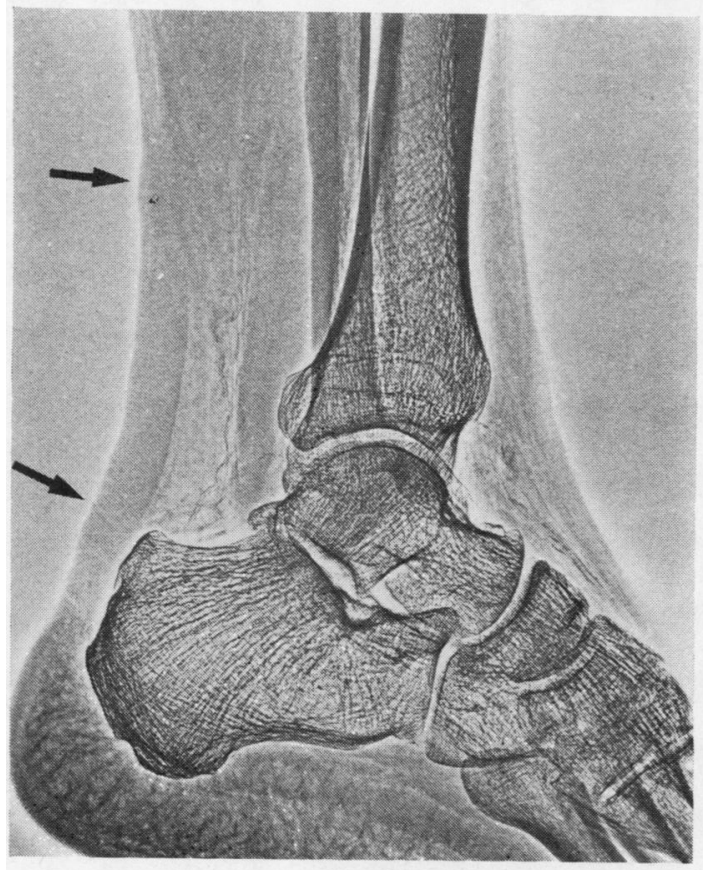

(b)

Case 9, male, aged 70, has suffered from chronic tophaceous gout since the age of 30 , with severe destruction of the hands and feet. In the past there were tophi on the hand, on the dorsal aspect of both feet, and in the Achilles tendons. Therapy with allopurinol, begun 10 years ago, arrested the process and the tophi eventually disappeared. Both Achilles tendons appeared normal to inspection and palpation, and were not tender. Xeroradiography (Fig. 9), shows a blurred and irregular aspect of the tendons but no tophus is apparent. 'Rat bite' erosions of the upper calcanei are seen.

The last case illustrates another unique lesion of

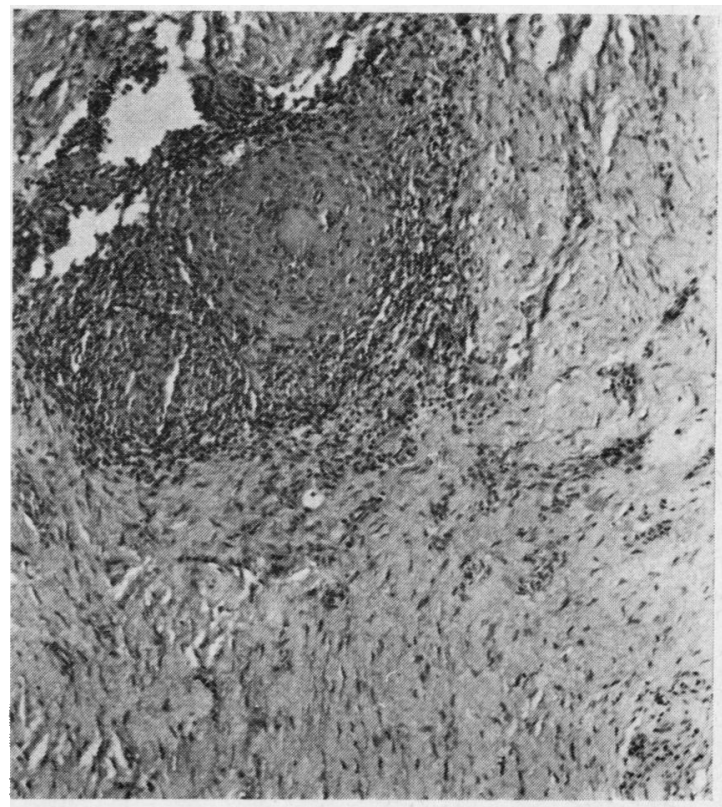

(c)

FIG. 5 Tuberculous peritendinitis (Case 5). (a) Right heel. (b) Xeroradiograph, showing a diffuse swelling with nodules (arrows) of the Achilles tendon; inferior diameter $9 \mathrm{~mm}$; diameter at middle part $10 \mathrm{~mm}$. (c) Photomicrograph of peritendinous area showing tubercle formation. Haematoxylin and eosin. $\times 65$

the tendon, as it can be exceptionally observed in patients with articular chondrocalcinosis.

Case 10, female, aged 82, had an 8-year history of repeated pseudogout attacks affecting both knees. Positive birefringent crystals were shown in the synovial fluid; radiologically, calcifications of the articular cartilages of the knees were present. The patient had suffered for 5 years from intermittent pain in the posterior part of the heels on walking. Pressure over the insertions of Achilles tendons was painful. On conventional $x$-rays, there were calcifications in the distal part of the left Achilles tendon (Fig. 10a), on xeroradiograph (Fig. 10b) these calcifications appear linear and are also seen in the right tendon. 


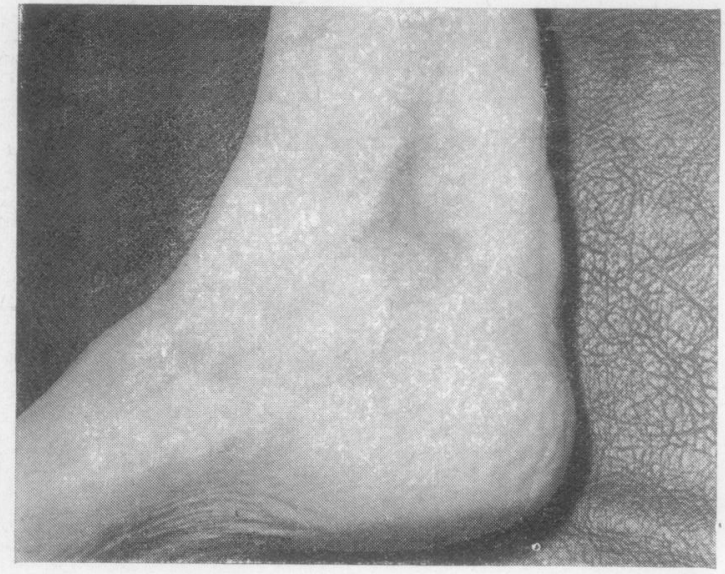

(a)
FIG.6 Tendinous hypercholesteraemic xanthomata(Case 6). (a) Right heel. (b) Xeroradiograph showing cylindric nodular thickening of both Achilles tendons. Diameter at insertion $6.0 \mathrm{~mm}$ at both sides. Diameter at middle part: right 12.0 $\mathrm{mm}$; left $18.0 \mathrm{~mm}$

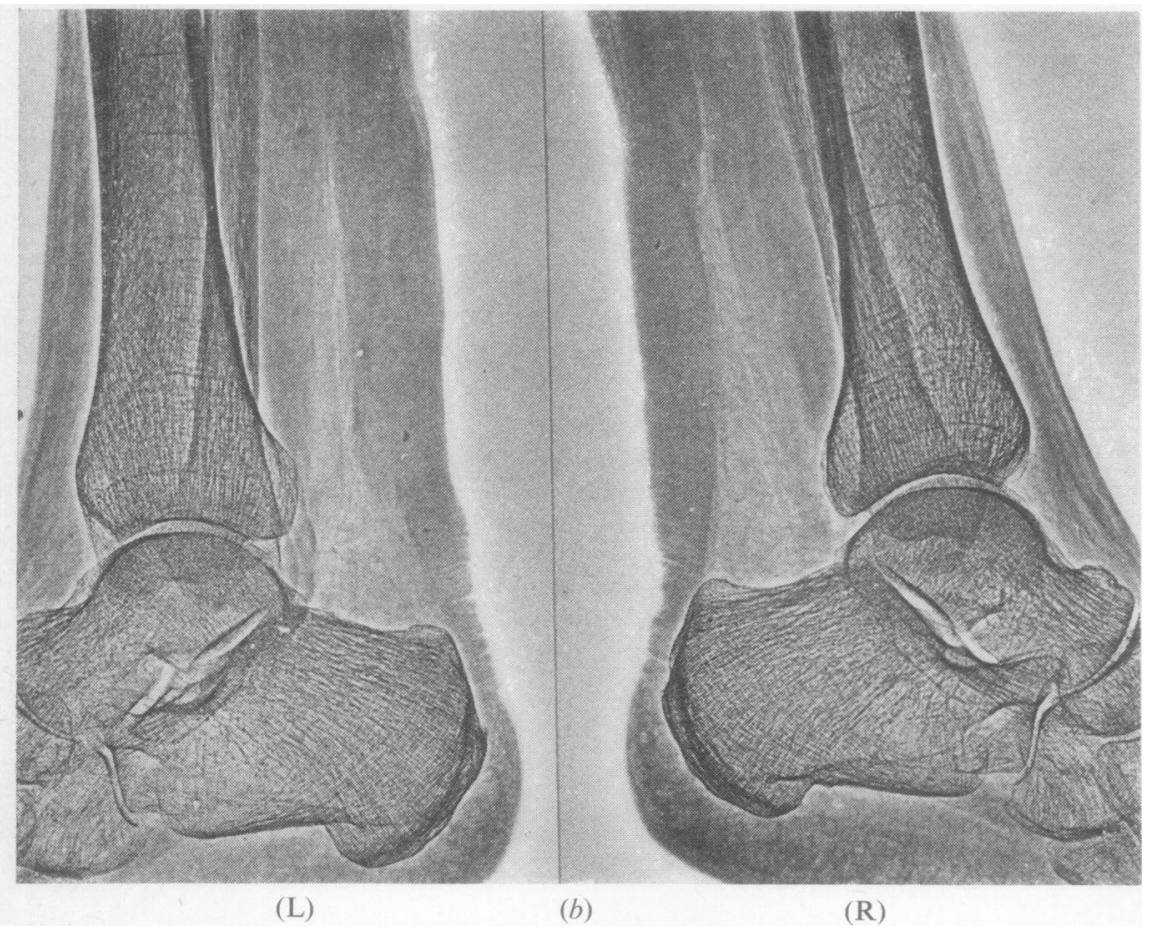

\section{Discussion}

In rheumatoid arthritis, ankylosing spondylitis, and Reiter's disease heel lesions can occur (Davis and Blair, 1950; Bywaters, 1954; Calabro, 1962; Brousse, Braun, Amor and Coste, 1966) and they sometimes represent the first signs of the disease presenting clinically as pain on the posterior aspect of the calcaneum, at the insertion of the Achilles tendon or on its plantar aspect, and at the insertion of the plantar aponeurosis.
The earliest lesions can be an Achilles peritendinitis (Fig. 2), an achillobursitis (inflammation of the subAchilles bursa) (Fig. 4a), or an inflammation at the attachment of the plantar aponeurosis (plantar fasciitis) (Fig. 3). These lesions involve the soft tissues and usually escape detection on conventional $x$-ray but become visible with special exposure. Xeroradiography permits detailed visualization of the soft tissues and also, on the same exposure, of the bone structure. Thus the erosions of the os calcis, the periosteal new bone formation, or the spurs which 


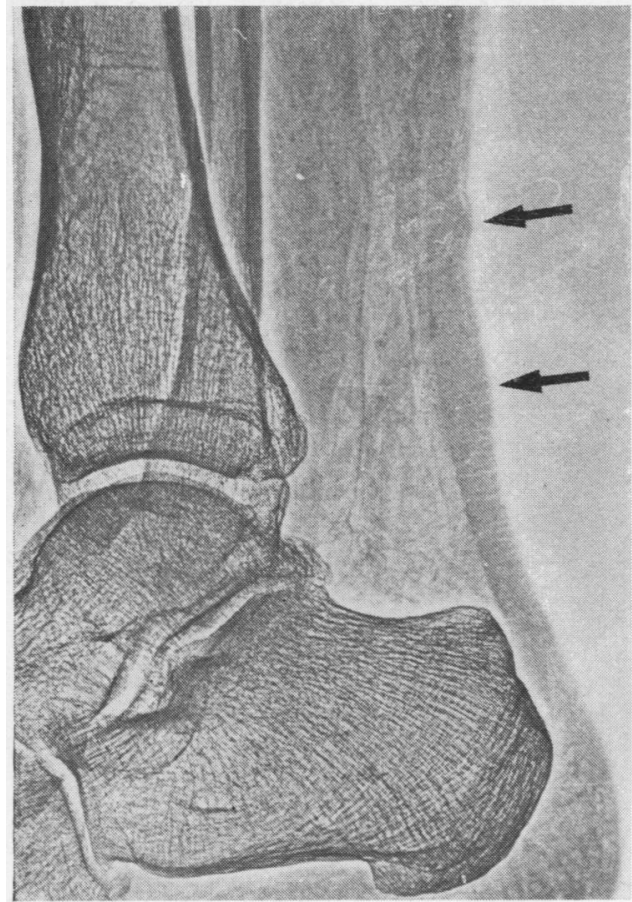

FIG. 7 Two tophi on the left Achilles tendon (arrows) (Case 7). Diameter of the superior nodule $7.5 \mathrm{~cm}$. Diameter of the inferior one $9.0 \mathrm{~mm}$

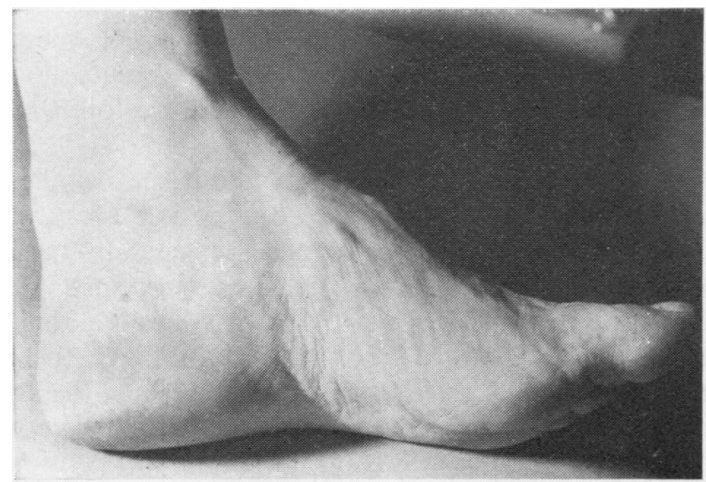

(a)

FIG. 8 Gout (Case 8). (a) Tophaceous nodule of the Achilles tendon. (b) Xeroradiograph

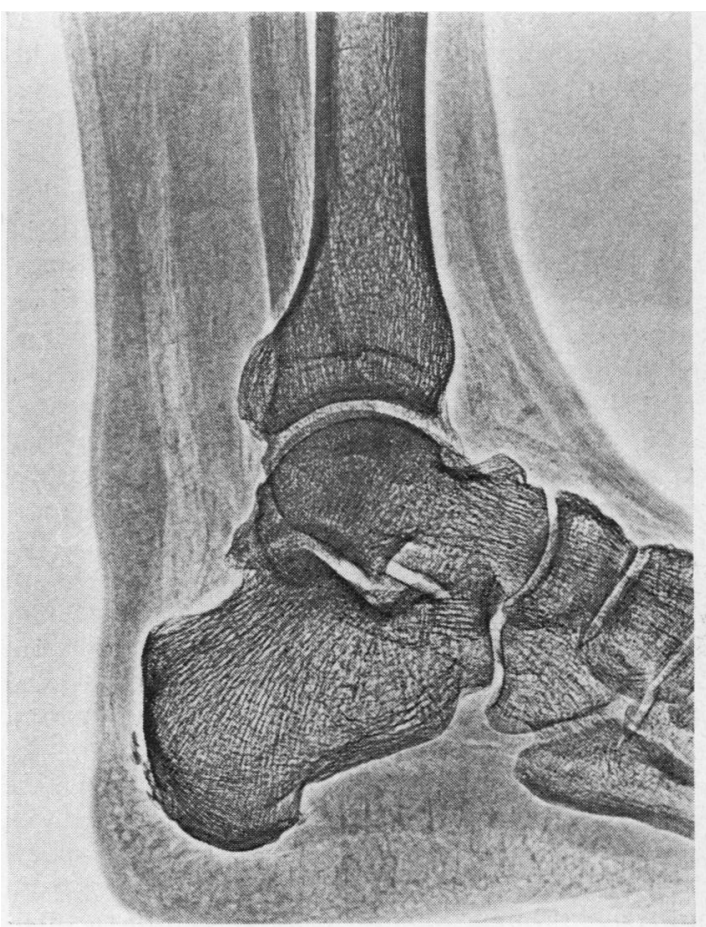

(b) 


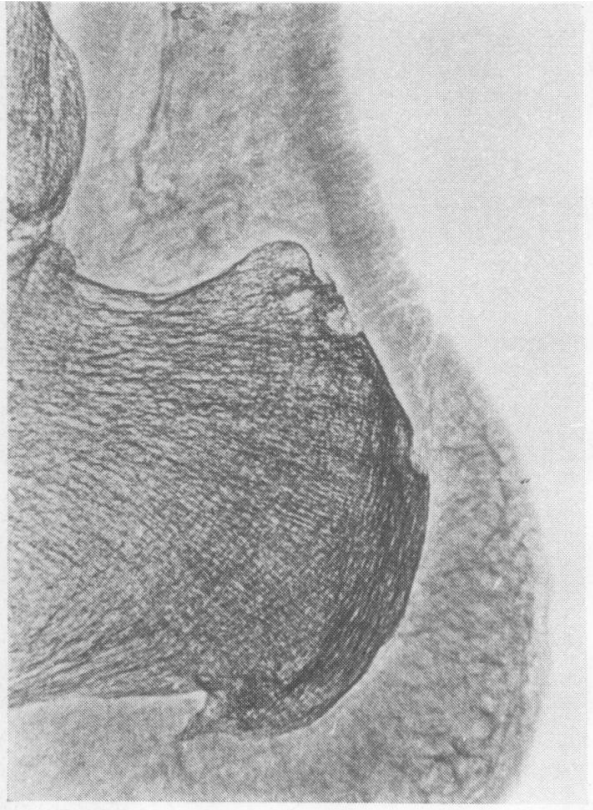

(L)

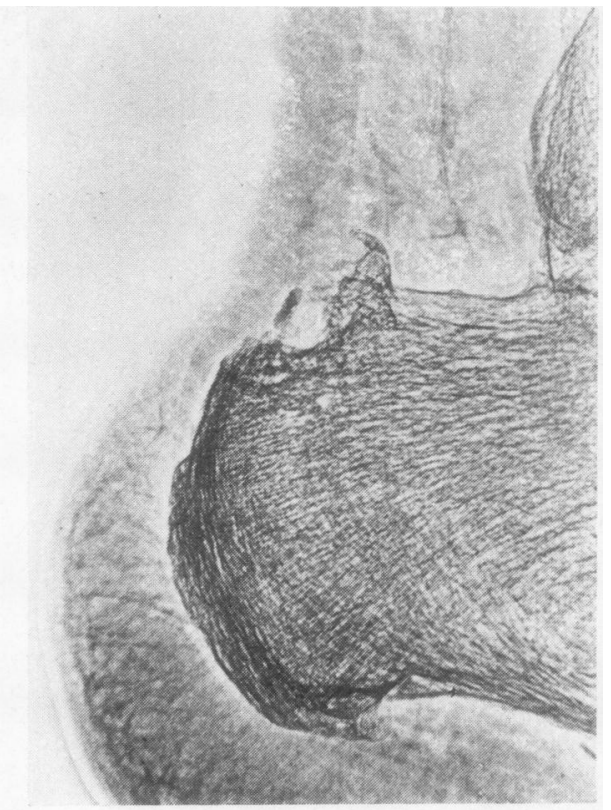

(R)

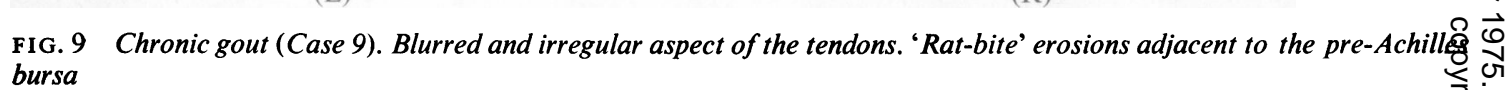

commonly accompany the natural progression of peritendinitis, achillobursitis, or plantar fasciitis can be seen on the same picture. The radiological changes of the calcaneum in rheumatoid arthritis, ankylosing spondylitis, and Reiter's disease have been well analysed by Mason, Murray, Oates, and Young (1959).

Tuberculous peritendinitis is a rare condition, generally involving flexor and extensor tendon sheaths of the hand and dorsiflexor tendon sheaths of the ankles (Garre, 1891 ; Mason, 1934; Adams, Jones, and Marble, 1940). A localization to the Achilles tendon seems quite exceptional and has been, to our knowledge, described only once before (Blenke, 1908). In our case, the turberculosis was probably favoured by the long-term treatment with prednisone and azathioprine for polymyositis. Xeroradiography gave a clear picture of the diffuse nodular peritendinitis (Fig. 5).

Xanthomatous deposits in the Achilles tendons are common in type II hyperlipoproteinaemia (March, Gilbert, and Kain, 1957; Fredrickson, Levy, and Lees, 1967). Generally, both tendons are thickened in a nodular, cylindrical fashion, which is apparent on the xeroradiograph of our patient (Fig. 6).

Tophi may be localized in the feet but rarely in the Achilles tendons, since only 10 cases were found in a total of 520 gouty subjects by Paillard and Fauvert (1945). In some cases destructive gouty erosions from pre-Achilles bursa, similar to those seen in rheumatoid arthritis are present (Fig. 9). Xeroradiography can detect clinically inapparent tophi (Fig. 7) or an irregular aspect of the tendons indicating a previous tophaceous involvement (Fig. 9). It is a simple and reliable radiological method for objective assessment of tendinous tophi and xanthomata, and regression of the nodules during treatment can be followed easily.

Little information is available about the Achilles tendons in articular chondrocalcinosis; Munthe, Pahle, and Reitan (1969, and unpublished data) have described 3 cases with calcium-pyrophosphate dihydrate crystal deposit in the Achilles tendons, proved by $x$-ray diffraction examination. In these cases, as in ours, the calcifications were fine, linear, lacking the rough aspect of a common calcaneal spur 윽 (Fig. 10a). These calcifications are more apparent on $D$ the xeroradiograph (Fig. 10b). This method gives a greater resolution of fine structures and accentuation $\mathrm{N}$ of the density of the calcifications. In this case it permitted the discovery of calcium pyrophosphate $O$ deposits in the right tendon, where they were not $\omega$ apparent with conventional techniques. These $\mathcal{O}$ tendinous deposits can produce a swelling of the distal part of the Achilles tendon and lead to chronic pain of the posterior heel.

We are grateful to Professor T. L. Vischer for help and $\overparen{D}$ advice in the writing of this paper; to Mr C. Menge and $\stackrel{\oplus}{\oplus}$ G. Rapin for technical assistance; and to Mrs Constantin $\varrho$ for secretarial service. 


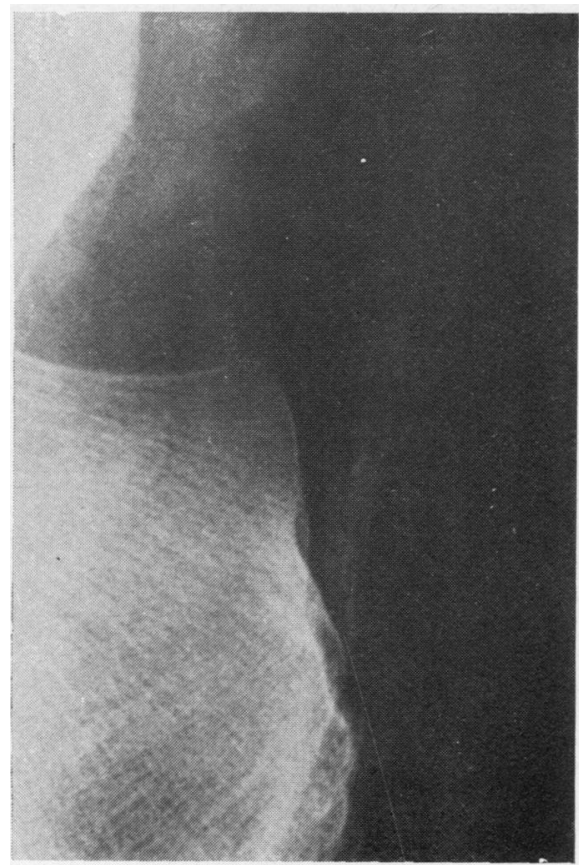

(L)

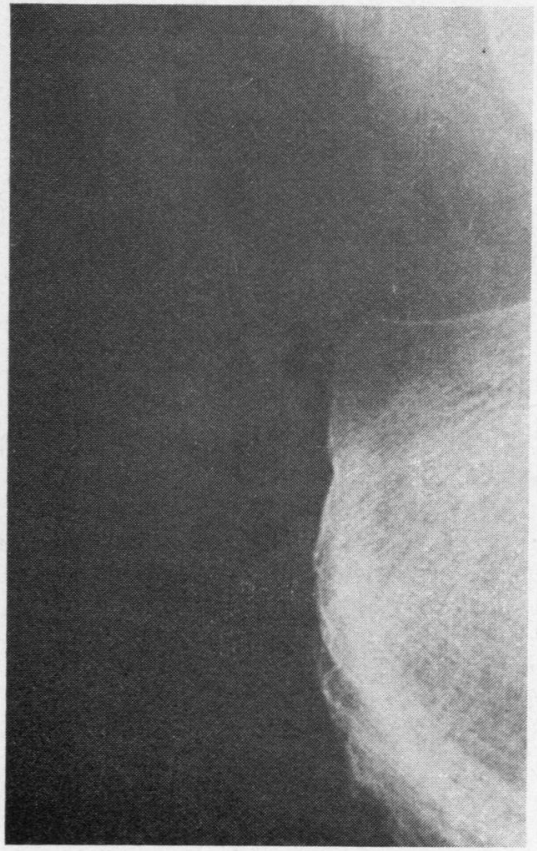

(R)

(a)

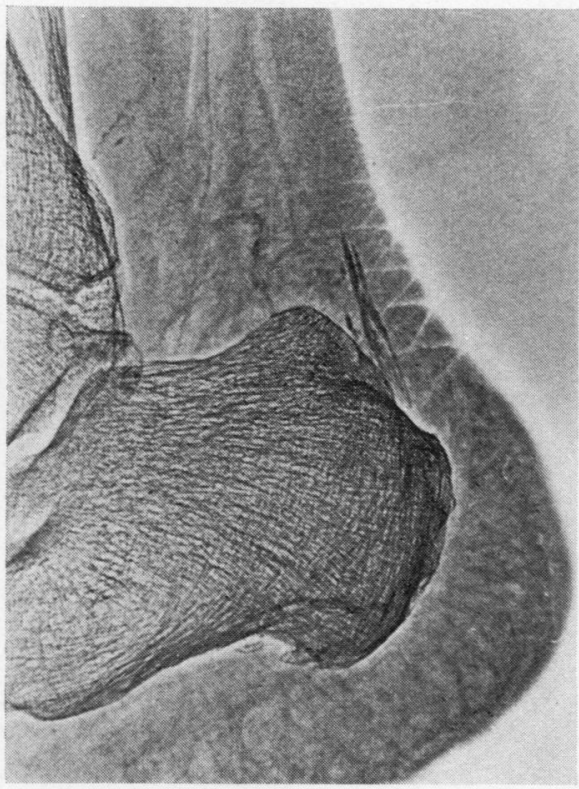

(L)

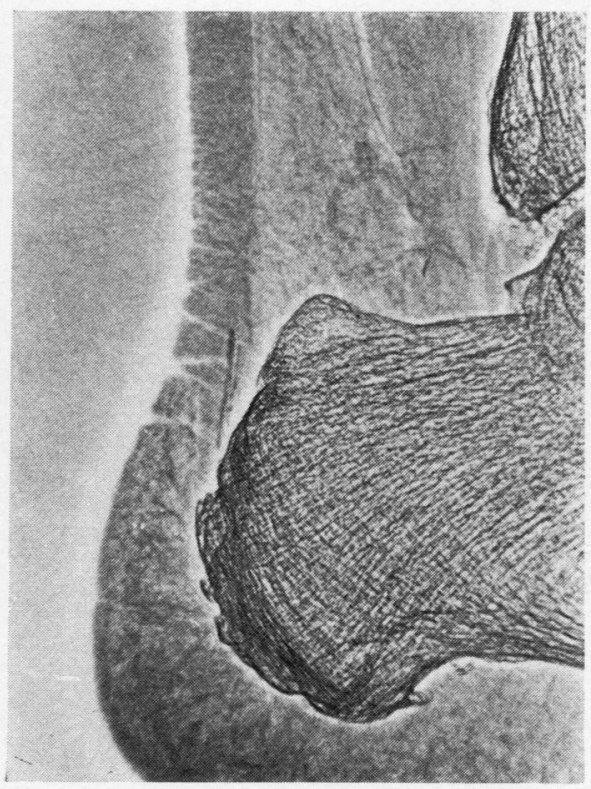

$(\mathrm{R})$

FIG. 10 Articular chondrocalcinosis (Case 10). Linear calcifications above attachment of the Achilles tendons. (a) Conventional $x$-ray technique. (b) Xeroradiography showing that the density of the calcification is accentuated; note a greater resolution of the fine structures. The inferior diameter of the left Achilles tendon is $9.0 \mathrm{~mm}$, right $5.0 \mathrm{~mm}$. Note a thickening of the left posterior heel pad.

(b) 


\section{References}

Adams, R., Jones, G., AND Marble, H. C. (1940) New Engl. J. Med., 223, 706 (Tuberculous tenosynovitis) BLENKE, A. (1908) Z. orthop. Chir., 20, 363 (Bemerkungen über den 'Calcaneussporn')

Brousse, J. P., Braun, S., Amor, B., ANd Coste, F. (1966) Sem. Hôp. Paris, 13, 795 (Etude comparative de quelques types de talalgie et de calcanéite)

Bywaters, E. G. L. (1954) Ann. rheum. Dis., 13, 42 (Heel lesions of rheumatoid arthritis)

CAlABro, J. J. (1962) Arth. and Rheum., 5, 19 (A critical evaluation of the diagnostic features of the feet in rheumatoid arthritis)

Davis, J. B., AND Blair, H. C. (1950) J. Bone Jt Surg., 32A, 838 (Spurs of the calcaneus in Strümpell-Marie disease)

Fredrickson, D. S., LeVy, R. I., AND LeES, R. S. (1967) New Engl. J. Med., 276, 215 (Fat transport in lipoproteins)

GARRE, C. (1891) Bruns' Beitr. klin. Chir., 7, 293 (Die primäre tuberkulöse Sehnenscheidenentzündung)

MARCh, H. C., Gilbert, P. D., AND KaIN, T. M. (1957) Amer. J. Roentgenol., 77, 109 (Hypercholesteremic xanthomata of the tendons)

Mason, M. L. (1934) Surg. Gynec. Obstet., 59, 363 (Tuberculous tenosynovitis of the hand: a study of 33 cases of chronic tenosynovitis of the hand)

Mason, R. M., Murray, R. S., OAtes, J. K., and Young, A. C. (1959) J. Bone Jt Surg., 41B, 137 (A comparative radiological study of Reiter's disease, rheumatoid arthritis and ankylosing spondylitis)

MunThe, E., PAHLE, J., AND Reitan, H. (1969) 'Studies in extra-articular calcifications in joint chondrocalcinosis and other calcinosis' in '12th Congressus Rheumatologicus Internationalis', Prague, Abst. 778. Geigy, Basle

Oliphant, W. D. (1955) Brit. J. Radiol., 28, 543 (Xeroradiography. Apparatus and method of use)

Paillard, H., AND Fauvert, R. (1945) 'Les tophi et les infiltrations tissulaires de la goutte', in 'La Goutte', p. 23. Baillière, Paris

WolfE, J. N. (1969) Radiology, 93, 583 (Xeroradiography of the bones, joints and soft tissues) 